very struck in the last year or so with the number of people with malignant disease of the proximal part of the large bowel who first presented with inflammatory complications (it did not happen in this case), but so many cases have come up and it has been difficult to decide whether the lesion was a carcinoma or an appendix abscess. There were two such cases in the ward recently, both old men, one turned out to have an appendix abscess and one was a carcinoma.

\title{
Mixed Salivary Tumour of Right Cheek
}

\section{Case History (Mr. W. Black)}

The patient, a married woman of 45 , was admitted for diagnosis and treatment of a lump in the cheek situated at the right angle of the mouth. This had been present for four years and had gradually increased in size causing reddening of the skin overlying it. For some months prior to admission it had been painful. There had been no ulceration or bleeding, and no difficulty with swallowing or speech. Her general health was good and on examination there was a hard, irregular swelling $2 \mathrm{~cm}$. in diameter in the cheek, just to the right of the mouth. The mucosal aspect was whitish and fissured, and the surrounding mucosa showed a few small leukoplakic patches. On the skin surface there was a small reddish, nodular area in the centre of the tumour, I $\mathrm{cm}$. in diameter. The tumour was confined to the cheek and did not extend to the alveolar mucosa.

A biopsy was performed within the mouth and section showed a mixed salivary tumour. Accordingly the patient was admitted for excision and plastic repair. At operation, by Mr. Ewing, it was evident that more mucous membrane would have to be removed than skin. An incision was made through the skin encircling the tumour, and this incision was deepened through the cheek to the mucous membrane, which was cut with scissors, keeping well clear of the growth. A bridge of the vermilion border of the lip was left, but the mucous membrane was removed from the cheek right to the alveolar margin. Haemostasis was secured and the defect was then closed by $\mathrm{Mr}$. Barron in the following way:-

The defect in the mucous membrane was closed by straight suture between the vermilion border of the lip and the cut edge of the mucous membrane along the alveolar margin. These sutures were of catgut and were tied on the buccal surface. A roughly triangular defect in the skin and soft tissues was left, the base of which extended from the angle of the lip downwards over the chin and the apex pointed towards the right

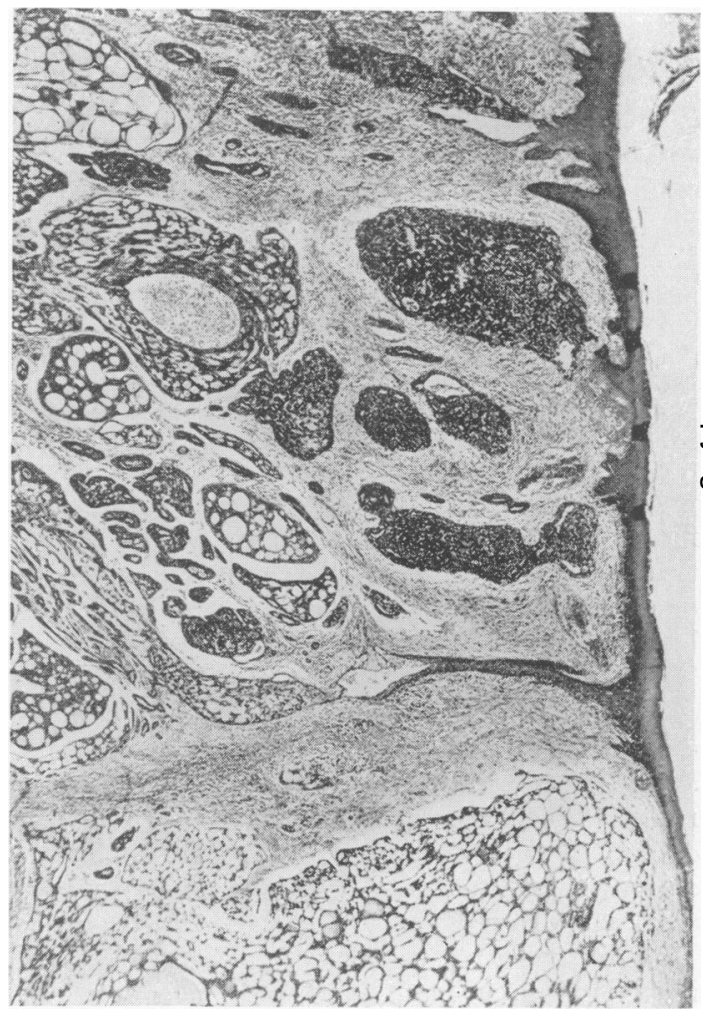

ear. Mr. Barron now fashioned a rotation flap by extending the base of the triangle along a wide arc over the cheek and neck. This incision was deepened to the platysma and the skin flap contained within it was rasied and the whole flap rotated upwards to cover the defect. A pressure dressing was applied.

Convalescence was uneventful, but a small area of the flap at the corner of the mouth sloughed and this will require further plastic procedures at a later date. 


\section{Pathology (Dr. Pearse)}

The specimen consists of a piece of skin 32 by $32 \mathrm{~mm}$. with an $8 \mathrm{~mm}$. white boss, separated by $18 \mathrm{~mm}$. from a piece of mucous membrane 24 by $14 \mathrm{~mm}$. Gross section shows a translucent, firm tumour 22 by $14 \mathrm{~mm}$., probably not completely removed on one side.

The histology is that of a mixed salivary tumour of so called cylindromatous type. It consists of epithelium arranged both in acini and in trabeculae in a hyalinized stroma.

\section{Discussion}

Prof. AIrd: I find these tumours very fascinating, not only in their normal sites but also in the abnormal situations in which they occur. They may be found either on the mucosa of cheek, in tongue, palate, gum, fauces and in the pharynx. I would like to have some authoritative statement about the behaviour of these extra-parotid tumours, if you can call them that, when compared with those which occur in the parotid gland itself. In the parotid gland we treat them both by surgery and by radiotherapy, so likely is it that some little remains of tumour may spread into or beyond the capsule, as here. We do not carry out here a formal excision of parotid gland, for we do not believe that the anatomy of the facial nerve is uniformly as it has been described. We adopt the form of treatment advocated by the Swedes, particularly Dr. Ahlbom. He does a Hybbinette operation, which involves scraping out the tumour, having let it ripen to about the size of a walnut. Then, putting in the tip of one's finger, one dissects out the capsule like the tip of a glove. Having done that we irradiate the gland. Ahlbom has shown that treatment of these tumours by irradiation alone is responsible for a recurrence rate of about 50 or 60 per cent. Surgery alone has about the same success, but a combination of both will give a cure rate of about 80 or 90 per cent. Do the tumours outside the parotid gland behave in precisely the same way? Tney seem to be more accessible to surgery in these areas but the cosmetic results in the face limit the ambition of a surgeon. In the tongue and in tne pharynx they are really easier to excise by the knife.

Mr. BRUCE DICK: I have been interested in the brother tumour to this (and it is essentially a brother), that is, the bronchial adenoma. I have carried away the impression from recent readings that the mixed parotid tumour is an essentially innocent tumour in that it does not metastasize. The mucous content is characteristic of this type of tumour and I understand it is radiosensitive to a high degree. What surprised me was that the surgeon took upon himself to be so drastic in re- moving a tumour like this. I have never heard of one in this location; it is a unique place for a parotid tumour.

Prof. Dible: I do not know what the fate of this tumour is outside the parotid. I have only seen it in the parotid and the nasal septum; in either situation the histology is very much the same. One point was brought out by Macfarlane in America, who reviewed a very large series of these tumours, and that is that the histological appearance is a very indefinite guide to the clinical behaviour of the tumour. He tried to guess the prognosis from the histological character and found very little correlation at all.

Mr. EwING: I would disagree with Mr. Dick. I think these tumours should be regarded very seriously and treated like malignant growths. If the parotid gland does not lend itself to radical resection I think we should call in the radiotherapeutists. If I have a mixed tumour of the parotid in the cheek I shall want to have the radical surgery described by Mr. Black.

Prof. AIRD: This tumour occurs also in the bronchus. What is the treatment of mixed tumour of the bronchus?

Mr. BRUCE Dick: I see women going about the streets with great big parotid tumours they have had for 30 or 40 years and they will not part with them. The tumours in the bronchus are entirely benign. It would seem to be accepted by pathologists that they belong to the same family and have the same histological structure, but mucous formation is exceptional. They enjoy exceptional immunity to this.

DR. Doniach: I thought, when I first saw it, that this tumour was an unusual type of basal cell carcinoma of the face, possibly arising from a sweat gland. These tumours look remarkably similar to salivary gland tumours. I wondered about the sensitivity to radiotherapy in this case. Was radiotherapy attempted after biopsy?

Mr. BlaCK: No. The biopsy report was benign salivary gland tumour.

Mr. BRUCE Dick: In these tumours has a metastasis ever been described?

Prof. Dible: I have never seen a gland bearing metastases, nor one at post-mortem. Since they pass out of our view what is the final state of these people we talk about?

Prof. AIRD: Mr. Patey wrote a paper about eight years ago from the Middlesex Hospital, and in that paper he quotes one person, I think, as having died of a metastasis from what was regarded as a benign mixed salivary gland tumour, but so rare appears to be that metastatic behaviour that I think the solitary case of a metastasis from one would really have to be very closely inspected 
histologically before that diagnosis was submitted.

Mr. EwING: We had a good photograph of a woman in this hospital who had a very large parotid tumour below the angle of the mandible, and who was treated by radiotherapy alone.

Prof. AIrd: The Swedes are very cautious in their classification of these mixed tumours and they describe three varieties; the benign variety, the potentially malignant variety and an intermediate variety (which includes 40 per cent. of all tumours); the criteria they adopt for malignancy are:-Cell type, nuclear type, extension beyond capsule, invasion of blood vessels and papillary formation. This particular tumour would be considered to have a good prognosis. These criteria they describe refer only to local malignancy as one would use the word in connection with a basal cell tumour, and I do not remember them describing a case of metastasis.

Mr. EwING: In Sweden I saw a Lapland woman with a mixed parotid tumour she had had for 20 years. It had grown to the size of her head and was tucked in a shawl, but of course we endeavour not to have people in this country walking around with tumours of this kind.

Prof. AIRD: If, as the Swedes did, you count up the years, taking the average number of years of duration of a benign salivary tumour, and of a malignant salivary tumour, they are almost identical. Benign are present for about nine years before they report for treatment, and malignant about seven years.

Mr. Peter Martin: It has been my feeling that these tumours must be treated seriously. I would be inclined to think that if it were feasible the parotid gland should be removed. I would certainly always try to remove some gland tissue with the capsule, and I have one very severe recurrence which was absolutely inoperable.

PROF. AIRD: When recurrence does happen the histological appearance is always very similar to the original tumour. However, the rapidity of tumour growth is always very much greater on each successive recurrence than it was in the original tumour. The reason seems to be that the original operation does interfere with the capsular arrangement of the tumour and makes local spread of any remnants easier. I am entirely satisfied that the treatment of these is by local excision and subsequent irradiation. I met the Edinburgh radiotherapist, Dr. McWhirter the other day. We treated a few tumours by this method about six years ago by inserting radium needles to get a very high local concentration of radiation and in none of these cases has there been recurrence. Miss Constance Wood here uses irradiation after the manner of Finsey, using glancing fields across the cheek after we have done the excision.

\section{Malignant Melanoma of Leg}

Case History (Mr. J. J. Shipman)

The patient, a woman aged 30 years, was admitted to this hospital with a pigmented tumour of the skin of the left leg. On questioning it appeared that a mole had been present there as long as she could remember. Two years ago this spot had begun to increase in size. She noticed in addition to it becoming larger in diameter it was also becoming raised above the surface of the adjacent skin. Four months prior to admission the growth was about the size of the nail of the little finger, and about this time she broke the surface of it with a finger nail; it then discharged some mauve-brown jelly, followed by a discharge of watery material and blood. Following this event the wound healed, but since then she had noticed an acceleration in the rate of growth. She had not noticed any lumps appearing elsewhere and she has no cough.

On examination of the left leg the tumour was seen just above the middle of the medial border of the left tibia (Fig. I). The surface of the tumour was raised $\mathrm{I} \mathrm{cm}$. above the surrounding skin. The re was a shallow constricting ring around the base of the growth suggesting pedunculation. The surface of the tumour was covered by silvery flakes divided by clefts into segments, and was mauve in colour. The tumour felt as though it contained fluid surrounding a solid core. The growth was limited to the skin and could be moved on the subcutaneous tissues. A small soft gland was found in the left groin and a radiograph of the chest was normal. There were no abnormal physical signs in the abdomen.

Biopsy excision was decided upon by $\mathrm{Mr}$. Franklin, and this was performed by Mr. Chris. The whole tumour was removed, together with an area of normal skin and an area of deep fascia. Post-operative progress from this operation was satisfactory and it was followed by a block dissection of the glands of the groin two weeks later. The excision of the block of tissues was performed through a $\mathrm{T}$-shaped incision and, in addition to the main mass, a gland was removed from the femoral 